\title{
Condición corporal y su relación con producción láctea, reproducción y perfil metabólico en vacas lecheras del trópico boliviano
}

\author{
Body condition and its relationship with milk yield, reproduction and metabolic \\ profile in dairy cows of the Bolivian tropics
}
Vanessa Carizi Cherobin ${ }^{1}$, Juan Pablo Garzón P.,3,5, Juan Pedro Alvarado M. ${ }^{3}$, Pablo Roberto Marini ${ }^{3,4}$

\section{RESUMEN}

El objetivo de este trabajo fue analizar la relación de la condición corporal con la producción de leche, la eficiencia reproductiva y el perfil metabólico posparto en vacas Holstein del trópico boliviano. Se utilizaron 16 vacas Holstein primíparas y multíparas con edades entre tres y siete años durante los primeros 150 días de la lactancia. La condición corporal de los animales fue mejor en el preparto en comparación con el posparto $(p<0.05)$. Sin embargo, no hubo diferencias significativas entre la condición corporal y la producción de leche por vaca/día, producción de leche acumulada, peso corporal e intervalo parto - primer servicio y parto - concepción. Los componentes del perfil metabólico (glucosa, aspartato aminotransferasa, alanina transaminasa, proteína total, albúmina y urea) no presentaron diferencias significativas por efecto de la condición corporal.

Palabras clave: eficiencia reproductiva; comportamiento productivo; perfil metabólico

\section{AbStract}

The aim of this study was to analyze the relationship of the body condition score (BCS) with milk yield, reproductive efficiency and the postpartum metabolic profile in Holstein cows of the Bolivian tropics. Sixteen primiparous and multiparous Holstein cows aged between three and seven years were monitored during the first 150 days of

\footnotetext{
${ }^{1}$ Universidad Autónoma Gabriel René Moreno (UAGRM), Bolivia

${ }^{2}$ Estación Experimental del Austro, Instituto Nacional de Investigaciones Agropecuarias, Gualaceo, Ecuador

${ }^{3}$ Centro Latinoamericano de Estudios de Problemáticas Lecheras (CLEPL). Casilda, Provincia de Santa Fe, Argentina

${ }^{4}$ Facultad de Ciencias Veterinarias, Universidad Nacional de Rosario, Rosario, Argentina

${ }^{5}$ E-mail:dr.jpgarzon@yahoo.com
}

Recibido: 29 de marzo de 2018

Aceptado para publicación: 17 de octubre de 2018 
lactation. The pre-partum BCS was better than during the post-partum period $(\mathrm{p}<0.05)$. However, there were no significant differences between BCS and daily milk yield, milk production in the first 150 days, body weight, calving to first service interval and calving to conception interval. The components of the metabolic profile (glucose, aspartate aminotransferase, alanine transaminase, total protein, albumin and urea) did not show significant differences due to BCS.

Key words: reproductive efficiency; productive behavior; metabolic profile

\section{INTRODUCCIÓN}

La ganadería es un sector clave en la economía de los países de América Latina tropical, pues ocupa una amplia fracción de los recursos de tierras con potencial productivo, constituyendo una importante fuente de generación de empleo y de alimentos para todos los estratos sociales (Holmann et al., 2004). La tendencia mundial hacia la producción de leche a partir de forrajes se sustenta en la competitividad internacional para la reducción de los costos de producción y la expansión de la frontera agrícola, que da una oportunidad a los países de la franja tropical para producir leche de calidad en forma competitiva (Ibarra, 2004).

El análisis del perfil metabólico se ha utilizado para investigar posibles relaciones entre la ingesta de energía dietética y la fertilidad en las vacas lecheras; sin embargo, no ha surgido un patrón claro hasta el momento (Wathes et al., 2007). La pérdida marcada de condición corporal entre los periodos secos y posteriores al parto se asocian a cambios desfavorables en la condición corporal durante la lactación temprana, lo que provoca aumento de la incidencia de enfermedades metabólicas y reproductivas posparto, la disminución de los niveles de colesterol total durante el primer mes de lactancia e intervalos más largos a la primera inseminación postparto en vacas Holstein (Kim y Suh, 2003). Cuando las vacas llegan al parto en situación de obesidad o de pobre condición física tienen mayor riesgo de presentar difi- cultad de parto, desarrollar trastornos metabólicos, tener menor producción de leche y menor eficiencia reproductiva, así como de desarrollar una gran movilización de reservas corporales posparto (Rennó et al., 2006). El objetivo de este trabajo fue analizar la relación de la condición corporal (CC) con la producción de leche (PL), la eficiencia reproductiva (ER) y el perfil metabólico postparto (PMPP) en vacas Holstein del trópico boliviano.

\section{Materiales y Métodos}

El trabajo fue desarrollado entre octubre de 2016 y noviembre de 2017 en la Lechería El Prado de la Universidad Autónoma Gabriel René Moreno (UAGRM), ubicada en el municipio de Warnes, capital de la provincia de Ignacio Warnes, al norte de Santa Cruz de la Sierra, Bolivia. La zona se caracteriza como tropical con estación seca en invierno, presenta una temperatura media de $23^{\circ} \mathrm{C}$, una precipitación anual de $1528 \mathrm{~mm}$ y se encuentra a 332 msnm (Gobierno Autónomo Departamental de Santa Cruz, 2016).

Se utilizaron 16 vacas primíparas $(\mathrm{n}=4)$ y multíparas $(n=12)$ Holstein con edades entre 3 y 7 años. Las vacas fueron destinadas al potrero de preparto cuando tenían siete meses de gestación o su producción estaba por debajo de 7 1/día.

La alimentación preparto y posparto del hato bovino en estudio se basó en el pastoreo libre sobre pastizales de Brachiaria 
brizantha cv. Marandu. El balanceado comercial a base de cascarilla de arroz y el silo de maíz era proporcionado en corrales colectivos, conforme se describe en el Cuadro 1. Las vacas que terminaban la lactancia quedaban en corrales destinados a las vacas secas, en donde recibían la suplementación en comederos y solo salían a pastorear por un tiempo de dos horas al día. Luego del parto ingresaban al lote de vacas en ordeñe en donde las horas de pastoreo aumentaba (dos horas por la mañana y dos horas por la tarde) y recibían la suplementación en los corrales luego de cada ordeñe (Cuadro 1). Las vacas recibieron, además, sal mineral ad libitum. Las vacas recibieron el manejo sanitario habitual del establecimiento, el cual consistía en desparasitaciones, baños contra garrapatas y moscas cada 20 días o dependiendo de la carga parasitaria, vacunaciones para fiebre aftosa, carbunco, rabia bovina y estomatitis vesicular, así como aplicación inyectable de vitaminas y minerales.

La condición corporal (CC) fue evaluada mediante el método de Edmondson et al. (1989) en la escala de 1 a 5, por una misma persona a los 60 y 30 días antes del parto, en el momento del parto, y a los 30 y 60 días posparto. El peso $(\mathrm{P}, \mathrm{kg})$ fue registrado en el día del parto, y a los 30 y 60 días posparto, utilizando una balanza mecánica (Fujihira Industry Company, Japón) con capacidad de $1000 \mathrm{~kg}( \pm 5 \mathrm{~kg})$.

Para determinar los perfiles metabólicos posparto (PMPP) se tomaron muestras de sangre de la vena coccígea en tubos al vacío sin anticoagulante antes del suministro de alimento matutino. Las muestras fueron colectadas cada 15 días a partir del día 15 posparto y hasta los 60 días posparto. El suero fue obtenido por centrifugación a $2100 \mathrm{x} g$ durante 10-20 minutos y almacenado en microtubos en congelación.

La determinación de glucosa $(\mathrm{G})$ en $\mathrm{mmol} / \mathrm{dl}$, aspartato aminotransferasa (AST) en $\mathrm{U} / \mathrm{l}$, alanina transaminasa (ALT) en $\mathrm{U} / \mathrm{l}$, proteína total $(\mathrm{P})$ en $\mathrm{g} / \mathrm{dl}$, albúmina (Alb) en $\mathrm{g} / \mathrm{dl}$ y urea (U) en mmol/dl se obtuvo a través de un analizador semiautomático de química sanguínea (Stat Fax ${ }^{\circledR} 1904$ Semi-Automatic Chemistry Analyzer, Awareness Technology, EEUU), en el laboratorio de química sanguínea del Hospital Escuela de Veterinaria de la UAGRM. Los reactivos utilizados fueron TECO, de procedencia norteamericana.

La producción de leche (PL) se determinó mediante pesaje de leche de los ordeños de la mañana y la tarde los días $15,30,45$ y 60 posparto, y posteriormente en forma mensual hasta los 150 días posparto, utilizando el medidor graduado en litros conectado a cada conjunto de ordeño. Para la evaluación de la eficiencia reproductiva se determinó el intervalo parto - primer servicio (IPPS), número de servicios por concepción y el intervalo parto-concepción (IPC).

Para cada variable en estudio se estimó la media aritmética y el error estándar (EE). Se analizaron los promedios totales y por grupos. Las 16 vacas primíparas y multíparas se ordenaron según su $\mathrm{CC}$ a los 30 días del preparto en dos grupos: $\mathrm{G} 1,<3.5(\mathrm{n}=7)$ y $\mathrm{G} 2$, e»3.5 $(\mathrm{n}=9)$. Para el análisis estadístico se aplicó el análisis de variancia (ANOVA) en un criterio de clasificación de pruebas de comparaciones múltiples de Tukey-Kramer HSD $(\mathrm{p}=0.05)$. Los análisis estadísticos fueron realizados en el programa JMP v. 5.0 para Windows (JMP $\left.{ }^{\circ}, 2003\right)$.

Resultados y Discusión

\section{Toda la Población}

Las concentraciones de glucosa en plasma se pueden usar para monitorear el balance de energía en las vacas, donde bajas concentraciones de glucosa durante el posparto estaría asociado con una disminución de la eficiencia reproductiva (Harrison et al., 1990).

El valor promedio de glucosa fue de $2.8 \pm 0.4 \mathrm{mmol} / 1$, siendo significativamente menor a los 30 días posparto en comparación 
Cuadro 1. Composición de la dieta basal ${ }^{1}$ de las vacas Holstein en el pre- y posparto

\begin{tabular}{lcccc}
\hline \multirow{2}{*}{ Ingrediente } & \multicolumn{2}{c}{ Preparto } & \multicolumn{2}{c}{ Posparto } \\
\cline { 2 - 5 } & MS (kg) & PC (\%) & MS (kg) & PC (\%) \\
\hline Cascarilla de soya & 0.9 & 12 & 2.2 & 12 \\
Balanceado comercial & 0.9 & 12 & 6.5 & 18 \\
Silo de maíz & 7.5 & 7 & 7.5 & 7 \\
Brachiaria brizantha cv. Marandu $^{2}$ & 2.0 & 10 & 4.3 & 10 \\
\hline Total & 11.3 & 9.75 & 20.5 & 11.25 \\
\hline
\end{tabular}

${ }^{1}$ MS: materia seca; PC: proteína cruda

${ }^{2}$ Consumo estimado al pastoreo

Cuadro 2. Valores de aspartato aminotransferasa (ASL) y alanina transaminasa (ALT) (media \pm error estándar, U/L) en el posparto de vacas Holstein en el trópico boliviano

\begin{tabular}{ccc}
\hline Días & AST & ALT \\
\hline 15 & $75.4 \pm 7$ & $14.5 \pm 3$ \\
30 & $68.3 \pm 7$ & $13.5 \pm 3$ \\
45 & $70.6 \pm 7$ & $18.4 \pm 3$ \\
60 & $76.7 \pm 7$ & $19.1 \pm 3$ \\
\hline
\end{tabular}

con el valor obtenido a los 60 días posparto ( $\mathrm{p}<0.05$; Figura 1$)$, aunque dentro de los valores normales de $2.50-4.16 \mathrm{mmol} / 1$ para bovinos de leche (Dukes y Swenson, 1987). Los resultados fueron similares a los reportados por Quinteros-Pozo et al. (2017) en vacas de la amazonia ecuatoriana $(2.72 \mathrm{mmol} / \mathrm{l})$, lo que puede estar indicando un bajo nivel de energía de los alimentos. Por otro lado, se reportan valores de glucosa inferiores en los estadios iniciales del posparto, indicando un estado de hipoglucemia, pero que en fechas posteriores se llegan a valores considerados como normales para vacas lecheras (Minervino et al., 2004; Barrera, 2010). En este sentido, Ceballos et al. (2002b) señala que el aumento en la concentración de glucosa en la segunda semana preparto refleja los cambios hormonales producto de una mayor gluconeogénesis y glicogenólisis; aspecto que no es suficiente para satisfacer la demanda de glucosa en el inicio de la lactancia; resultados que coinciden con los de Margolles (1983) quienes indican que el ganado al pastoreo no sufre mayores cambios en la glucemia durante la lactación.

Los valores promedio de proteína total $(7.1 \mathrm{~g} / \mathrm{dl})$ y albúmina $(2.8 \mathrm{~g} / \mathrm{dl})$ no presentaron diferencias significativas entre muestreos y estuvieron dentro de los valores normales para vacas lecheras (Merck, 2000). No obstante, estos valores estuvieron por debajo de los reportados por otros autores (Saut, 2008; Barrera, 2010; Zarate et al., 2016). Por otro lado, Oliveira (2011) reporta valores de proteína total en el puerperio por debajo de los valores normales para vacas mestizas. Los valores promedio en el caso de la albúmina fueron similares a los reportados por Barrera, (2010) y Zárate et al. (2016), pero inferiores a los valores obtenidos por Saut (2008). Oliveira (2011), por otro lado, encontró valores bajos de albúmina a los 14 días del parto $(2.3 \pm 0.3 \mathrm{~g} / \mathrm{dl})$.

Los indicadores de función hepática (Cuadro 2) presentan valores dentro de los rangos aceptables (Merck, 2000). Si bien, 
Cuadro 3. Promedios y desvíos estándar de las variables metabólicas proteicas y energéticas según condición corporal $(\mathrm{G} 1:<3.5, \mathrm{G} 2:=3.5)$ en el periodo pre-y posparto de vacas Holstein en el trópico boliviano

\begin{tabular}{lcccccc}
\hline & $\begin{array}{c}\text { Glucosa } \\
(\mathrm{mmol} / \mathrm{l})\end{array}$ & $\begin{array}{c}\text { AST } \\
(\mathrm{U} / \mathrm{L})\end{array}$ & $\begin{array}{c}\text { ALT } \\
(\mathrm{U} / \mathrm{L})\end{array}$ & $\begin{array}{c}\text { Proteína } \\
(\mathrm{g} / \mathrm{dl})\end{array}$ & $\begin{array}{c}\text { Albumina } \\
(\mathrm{g} / \mathrm{dl})\end{array}$ & $\begin{array}{c}\text { Urea } \\
(\mathrm{g} / \mathrm{dl})\end{array}$ \\
\hline G1 (7) & $2.9 \pm 0.2^{\mathrm{a}}$ & $16.2 \pm 2.1^{\mathrm{a}}$ & $78.9 \pm 7.1^{\mathrm{a}}$ & $7.4 \pm 0.2^{\mathrm{a}}$ & $3.9 \pm 0.1^{\mathrm{a}}$ & $2.9 \pm 0.3^{\mathrm{a}}$ \\
$\mathrm{G} 2(9)$ & $2.9 \pm 0.1^{\mathrm{a}}$ & $17.4 \pm 1.8^{\mathrm{a}}$ & $70.2 \pm 6.1^{\mathrm{a}}$ & $6.8 \pm 0.2^{\mathrm{a}}$ & $3.9 \pm 0.1^{\mathrm{a}}$ & $2.8 \pm 0.3^{\mathrm{a}}$ \\
\hline
\end{tabular}

Letras iguales dentro de columnas indican ausencia de significancia estadística

ALT no es específica del hígado, su determinación es necesaria para diferenciar las alteraciones hepáticas de las musculares, situación que puede estar presente en bovinos, especialmente en el posparto. Los valores de AST fueron inferiores a resultados encontrados en la literatura científica (Galvis et al., 2003; Barrera, 2010; Penna Junior, 2010; Oliveira, 2011; Zárate et al., 2016), aunque similares a otros (Ceballos, 2002a; Lago et al., 2004). Wittwer (2000) considera que hay normalidad para valores debajo de $120 \mathrm{U} / \mathrm{L}$, mientras que para valores superiores podrían ocurrir lesiones hepáticas secundarias o excesivas movilizaciones lipídicas.

Los valores de urea se mantuvieron constantes, aunque bajos, durante el estudio, posiblemente debido a baja calidad de los pastos que no logran suplir las necesidades proteicas (Minervino et al., 2004; Nozad et al., 2012). Los valores obtenidos están por debajo de los mencionados por Ceballos (2002a) y Barrera (2010), aunque concuerdan con los resultados de Quinteros-Pozo et al. (2017).

La CC mostró una clara merma en el posparto comparado con el preparto, pero con tendencia a ir mejorando durante el posparto (Figura 2). Menegoto et al. (2015) señala que el $62 \%$ de las vacas pierden CC entre el periodo preparto y posparto. Los datos, en ge- neral, concuerdan con Franco (2015), que utilizó vacas mestizas Gir y Holstein con producciones de $18 \mathrm{l} / \mathrm{vaca}$ /día, pero mejores que los encontrados por Rennó et al. (2006), Freitas Júnior et al. (2008) y CedeñoQuevedo et al. (2011).

Los resultados de producción de leche por vaca/día no presentan diferencias significativas en el tiempo (Figura 3), como lo reporta Cedeño-Quevedo et al. (2011). La producción de $22.0 \pm 7.2 \mathrm{~kg} / \mathrm{vaca} /$ día en el pico de lactancia se encuentra dentro del rango comentado por Franco (2015), aunque superior al obtenido por Ortiz y Gaona (2016).

Con respecto al comportamiento reproductivo, todas las vacas se encontraban en el proceso de involución uterina normal a los 30 días del parto, no presentando signos de celo. El 12.5\% de los animales presentaron celo visible a los 60 días del parto, habiéndose realizado la inseminación artificial. El promedio general del IPPS fue de $109.3 \pm$ 41 días, el número de servicios por concepción fue de $1.8 \pm 1.0$ y el IPC fue de $147.9 \pm$ 65.8 días. Ocho vacas preñaron al primer servicio, representando $50 \%$ de todos los animales, valores encontrados por Ramos (2016) en vacas Holstein en sistema semi-intensivo, y mejores que los valores encontrados por Villadiego et al. (2016). 


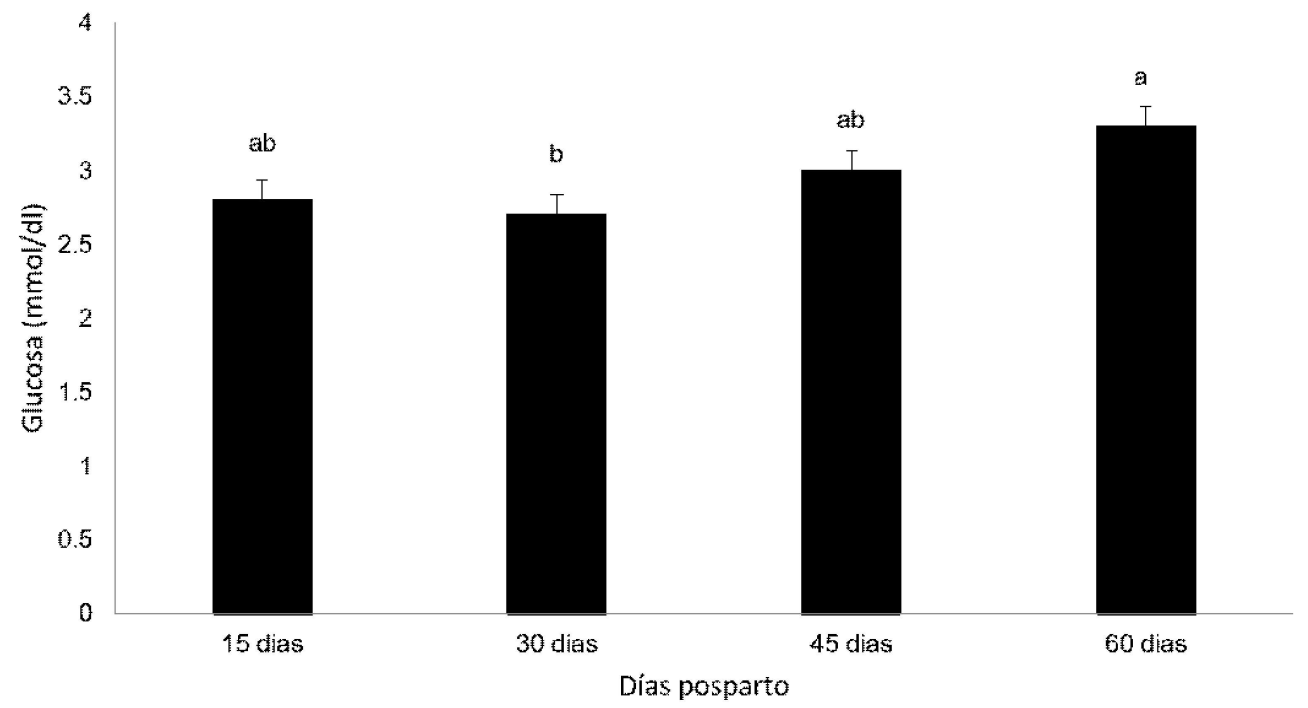

Figura 1. Valores de glucosa (media y error estándar) en el posparto de vacas Holstein en el trópico boliviano. Letras diferentes entre columnas indican diferencia significati$v a(p<0.05)$

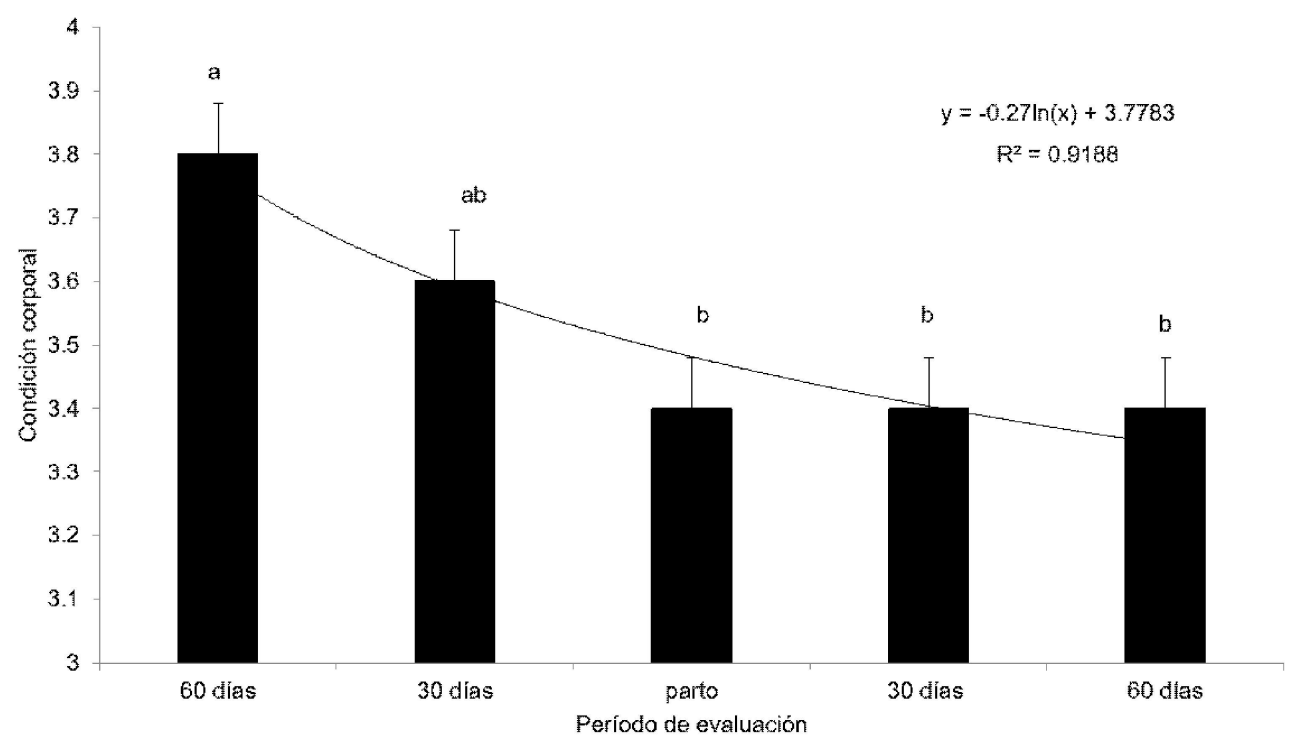

Figura 2. Condición corporal en el preparto y postparto de 16 vacas Holstein en el trópico boliviano. Letras diferentes entre columnas indican diferencias significativas $(p<0.05)$. 


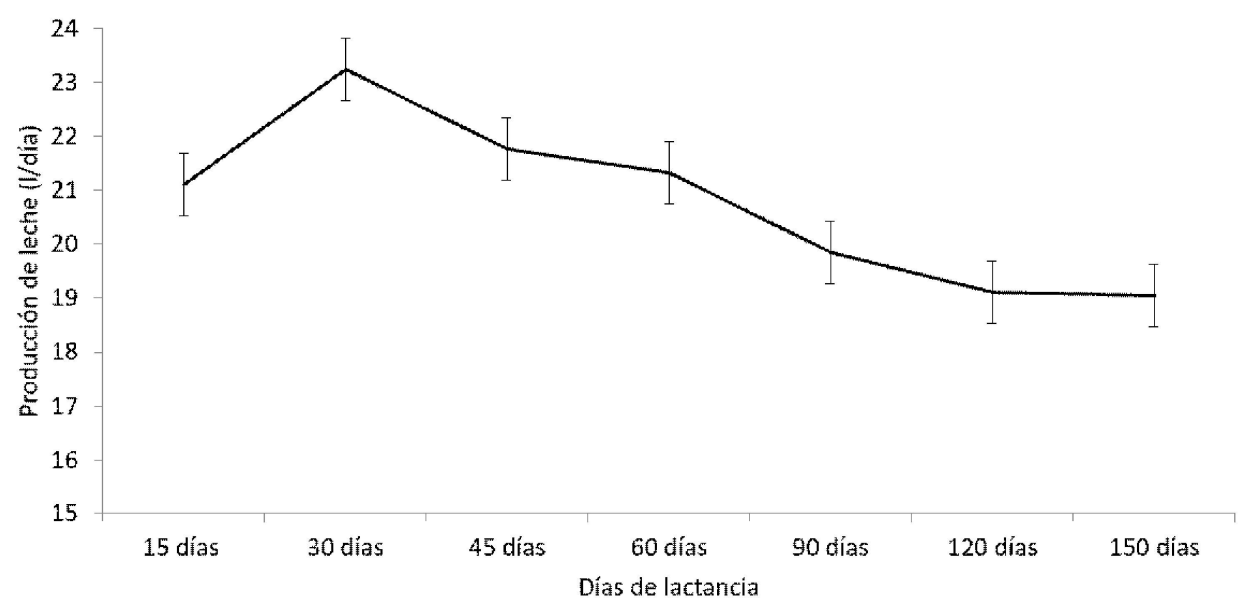

Figura 3. Producción de leche (1/vaca/día) de 16 vacas Holstein en el trópico boliviano

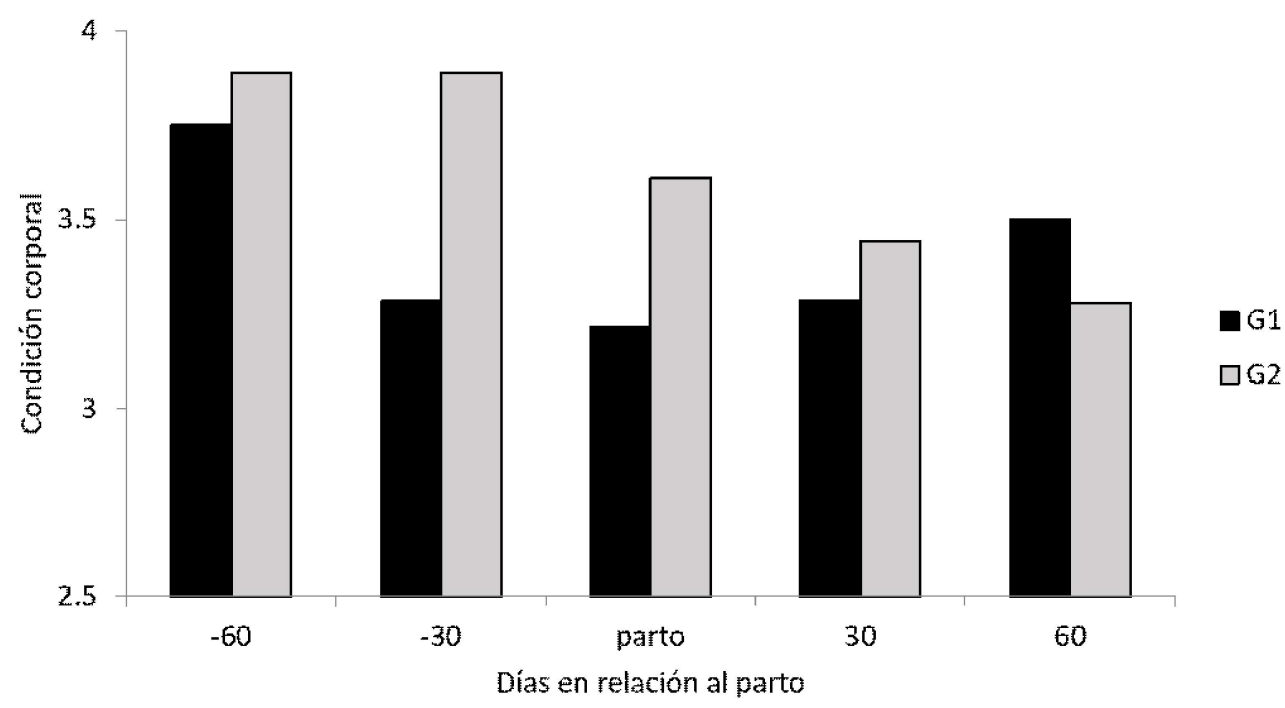

Figura 4. Condición corporal promedio y por grupo de condición corporal $(\mathrm{G} 1:<3.5, \mathrm{G} 2: \geq 3.5)$ en el periodo pre-y posparto de vacas Holstein en el trópico boliviano

\section{Por Grupos de Vacas}

Se encontraron diferencias significativas entre grupos de CC (Figura 4). Sin embargo, la CC registrada no afectó la producción de leche ajustada a los 150 días (G1: $2869 \pm 200 \mathrm{~L} ; \mathrm{G} 2: 3199 \pm 187 \mathrm{~L})$, la producción diaria de leche (G1: 19.1 $\pm 1.3 \mathrm{~L} ; \mathrm{G} 2: 21.3 \pm 1.3$ L), el peso vivo (G1:521 $\pm 12 \mathrm{~kg} ; \mathrm{G} 2: 518 \pm 10$ $\mathrm{kg}$ ), ni el intervalo parto - primer servicio (G1: $116 \pm 15$ días; $\mathrm{G} 2: 103 \pm 14$ días).
Las mayores pérdidas de $\mathrm{CC}$ ocurren en el periodo seco y en el puerperio (Fernandes et al., 2016). Ceballos et al. (2002b) observó una disminución en la CC hasta la semana seis del posparto, tanto en vacas de baja como de alta producción, coincidiendo con los resultados de G2 en el presente estudio. 


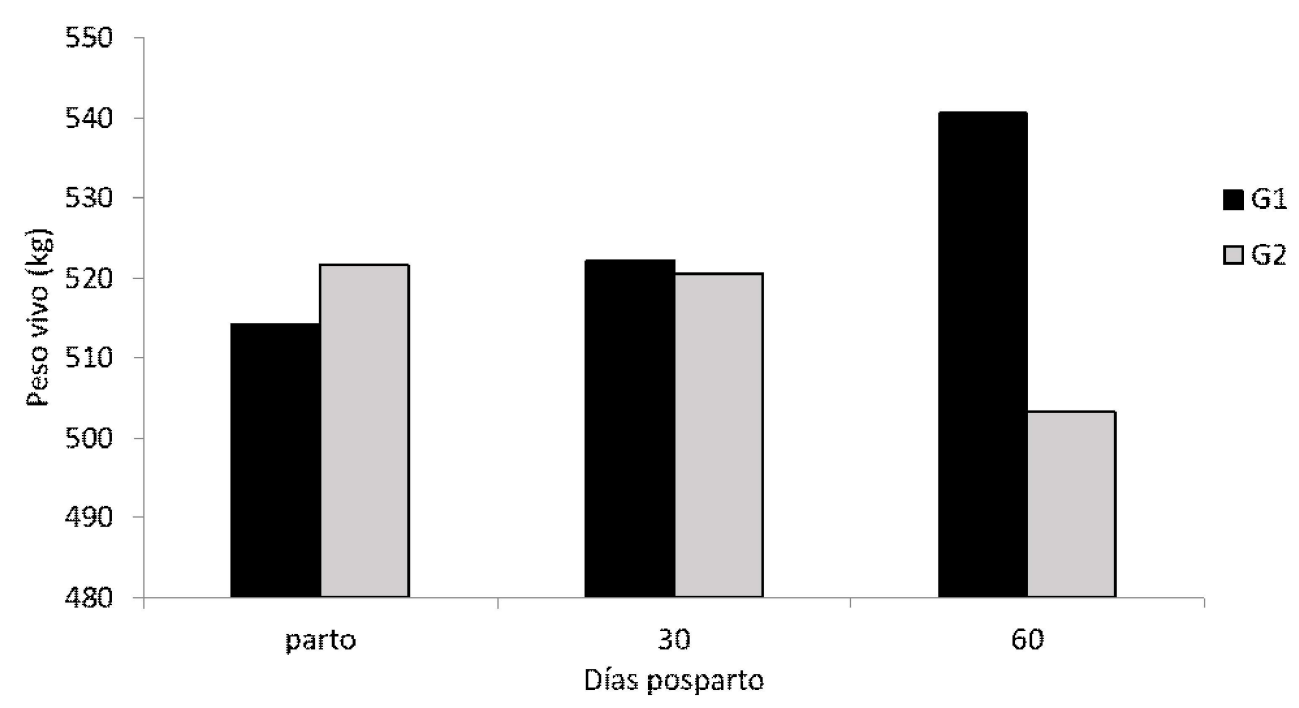

Figura 5. Peso vivo promedio y por grupo de condición corporal $(\mathrm{G} 1:<3.5, \mathrm{G} 2$ : $\geq 3.5)$ en el periodo pre-y posparto de vacas Holstein en el trópico boliviano

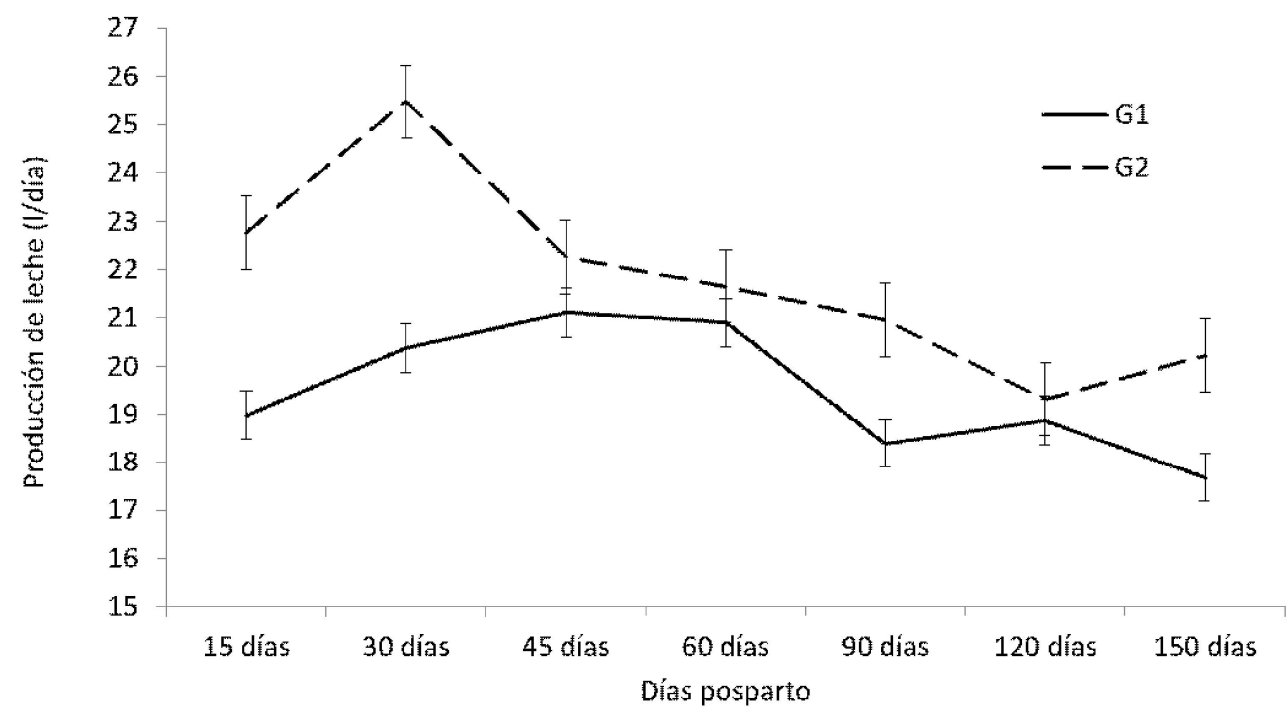

Figura 6. Producción de leche (1/vaca/día) según condición corporal (G1: $<3.5, \mathrm{G} 2: \geq 3.5)$ en el periodo pre-y posparto de vacas Holstein en el trópico boliviano 
Con relación al peso vivo a los 60 días posparto, G2 presentó mayores pérdidas de peso que G1 (Figura 5). Maza et al. (2006) menciona que la $\mathrm{CC}$ afectó la variación del peso postparto $(\mathrm{p}<0.10)$, pero no influyó sobre la producción de leche (0.10). Así mismo, observó que las vacas que paren con $\mathrm{CC}$ alta presentan pérdidas de pesos mayores y por periodos más prolongados. Oliveira (2002) reportó una pérdida de peso de $29.5 \mathrm{~kg}$ en las seis primeras semanas (558.2 $\mathrm{kg}$ de peso inicial).

Los valores de producción de leche según la $\mathrm{CC}$ se presentan en la Figura 6. La curva de lactación presentó su mayor valor en la semana 2 para G2 para luego descender gradualmente hasta los 150 días del estudio, mientras que la curva de lactación en G1 no sufrió mayores variaciones. No obstante, Oliveira (2002) observó un aumento gradual de la producción hasta la sexta semana posparto. Los resultados coinciden con los trabajos de Lago et al. (2001), Galvis et al. (2003) y Rennó et al. (2006), pero son superiores a los presentados por Freitas Júnior et al. (2008) y por debajo de los descritos por Oliveira (2002) hasta la sexta semana posparto. La producción de leche al inicio de la lactación ocurre, esencialmente, en función de las reservas corporales acumuladas en el preparto (Oliveira, 2002).

Franco (2015) menciona que las vacas con producción de leche mayor o igual a 24 $\mathrm{kg} /$ día tuvieran mayor pérdida de CC después del parto con relación a las vacas de producción igual o inferior a $17 \mathrm{~kg} /$ día. Salgado et al. (2008) encontraron una correlación positiva entre la variación de condición corporal y producción de leche $(\mathrm{r}=0.81$; $\mathrm{p}<0,05)$ en vacas doble propósito con $\mathrm{CC}$ de 4 al parto, mientras que Ceballos et al. (2002b) no encontraron asociación significativa entre estas variables.

El análisis de perfil metabólico no presentó diferencias significativas entre los dos grupos CC (Cuadro 5). Algunos análisis con- cuerdan con los valores encontrados por Michailoff et al. (2013) y Mancuso et al. (2016), pero están más elevados que los presentados por Campos et al. (2007), Noro et al. (2011) y Roa-Vega et al. (2017).

Ninguna vaca presentó celo a los 30 días del posparto. Solo una vaca de cada grupo de CC mostró signos de celo y fue inseminada dentro de los 60 días del posparto. El IPPS para G1 fue de 116.0 y para G2 de 103.4 días sin mostrar diferencias significativas entre grupos. El número de servicios por concepción fue de 1.86 y 1.75 para G1 y G2, respectivamente, y el IPC fue de 147.3 días y 148.3 días para G1 y G2, respectivamente, sin diferencias significativas entre grupos. De las 16 vacas en estudio, 13 quedaron preñadas (G1: 5 y G2: 8). Oliveira (2002) detectó el primer celo a los 69.5 días posparto en las 165 vacas Holstein de su estudio, con el suministro de una suplementación energética, así como 2.2 servicios por concepción y un IPC promedio de 172.6 días para el grupo control. Andrade (2013) indica que uno de los factores que influyen en un mayor IPPS es la baja condición corporal.

Santos et al. (2010) agruparon sus animales en función del cambio de la $\mathrm{CC}$ al inicio de lactación, encontrando que la tasa de concepción al primer servicio fue de $53 \%$ en vacas que movilizaron entre 0.5 y 1.0 unidad de CC y de $17 \%$ en aquellas que movilizaran más de 1.0 unidad de CC. Así mismo, mencionan que vacas que movilizaron mayores cantidades de reservas corporales presentaron mayores intervalos parto - primer celo, independientemente de sus niveles de producción de leche.

\section{Conclusión}

Los valores de condición corporal preparto de las vacas del estudio no afectaron la producción de leche, el comportamiento reproductivo ni su perfil metabólico. 


\section{Literatura Citada}

1. Andrade S. 2013. Desempenho produtivo e reprodutivo de vacas Holstein-Frísia em comparação com os respectivos cruzamentos com vermelha sueca e Montbéliarde. Tese de Mestrado. Lisboa, Portugal: Univ. de Lisboa. $76 \mathrm{p}$.

2. Barrera A. 2010. Avaliação metabólica de vacas leiteiras submetidas a diferentes estratégias de prevenção do balanço energético negativo no pós-parto. Tese de Mestrado. Brasil: Univ. Federal do Rio Grande do Sul. 66 p.

3. Campos R, Cubillos C, Rodas AG. 2007. Indicadores metabólicos en razas lecheras especializadas en condiciones tropicales en Colombia. Acta Agronómica 56: 85-92. doi: 10.15446/acag

4. Ceballos A, Villa NA, Bohórquez A, Quiceno J, Jaramillo M, Giraldo G. 2002a. Análisis de los resultados de perfiles metabólicos en lecherías del trópico alto del eje cafetero colombiano. Rev Colomb Cienc Pec 15: 26-35.

5. Ceballos A, Gómez PM, Vélez ML, ViIla NA, López LF. 2002b. Variación de los indicadores bioquímicos del balance de energía según el estado productivo en bovinos lecheros de Manizales, Colombia. Rev Colomb Cienc Pec 15: 13-25.

6. Cedeño-Quevedo DA, CeballosMárquez A, Garzón C, Daza-Bolaños CA. 2011. Estudio comparativo de perfiles metabólicos minerales en lecherías de dos regiones de Nariño. Orinoquia 15: 160-168.

7. Dukes HH, Swenson MJ. 1987. Fisiología de los animales domésticos. Tomo I y II. Ed. Aguilar. 1841 p.

8. Edmondson AJ, Lean IJ, Weaver LD, Farver T, Webster G. 1989. A body condition scoring chart for Holstein dairy cows. J Dairy Sci 72: 68-78. doi: 10.3168/ jds.S0022-0302(89)79081-0
9. Fernandes AFA, Oliveira JA, Queiroz $S A$. 2016. Escore de condição corporalen ruminantes. Ars Veterinaria 32: 5566. doi: 10.15361/2175-0106.2016v32n1p55-66

10. Franco FF. 2015. Escore de condição corporal e desempenho reprodutivo de vacas leiteiras mestiças lactantes. Tese de Mestrado. Minas Gerais, Brasil: Univ. Federal de Uberlândia. 57 p.

11. Freitas J., Rocha V, Rennó F, Mello M, Carvalho A, Caldeira L. 2008. Efeito da condição corporal ao parto sobre o desempenho produtivo de vacas mestiças Holandês x Zebu. Rev Bras Zootec 37: 116-121. doi: 10.1590/S151635982008000100017

12. Galvis RD, Correa HJ, Ramírez N. 2003. Interacciones entre el balance nutricional, los indicadores del metabolismo energético y proteico y las concentraciones plasmáticas de insulina, e IGF1 en vacas en lactancia temprana. Rev Colomb Cienc Pec 16: 237-248.

13. Gobierno Autónomo Departamental de Santa Cruz. 2016. Datos generales Municipio de Warnes. http://www.sicsantacruz.com/sic/index.php/boletines/pronosticos-meteorol $\% \mathrm{C} 3 \% \mathrm{~B} 3$ gicos

14. Gómez L, Campos R. 2016. Control del balance energético negativo y comportamiento productivo y metabólico en vacas doble propósito bajo suplementación energética. Rev Invest Agraria Ambiental 7: 147-156. doi: 10.22490/21456453.-1545

15. Harrison RO, Ford SP, Young JW, Conley AJ, Freeman AE. 1990. Increased milk production versus reproductive and energy status of high producing dairy cows. J Dairy Sci 73: 2749-2758. doi: 10.3168/jds.S00220302(90)78960-6

16. Holmann F, Rivas L, Carulla J, Rivera B, Giraldo LA, Guzmán $S$, Martínez M, et al. 2004. Producción de leche y su relación con los mercados: caso Colombiano. En: X Seminario de Pastos y Forrajes. Venezuela. 
17. Ibarra $A A$. 2004. Sistema de pagamento do leitepor qualidade, visão global. En: VII Congresso Brasileirode Qualidade do Leite. Curitiba, Brasil.

18. Kim IH, Suh GH. 2003. Effect of the amount of body condition loss from the dry to near calving periods on the subsequent body condition change, occurrence of post partum diseases, metabolic parameters and reproductive performance in Holstein dairy cows. Theriogenology 60: 1445-1456. doi: 10.1016/S0093-691X(03)00135-3

19. Lago EP, Pires AV, Susin I, de Faria $V P$, Lago LA. 2001. Efeito da condição corporal ao parto sobre alguns parâmetros do metabolismo energético, produção de leite e incidência de doenças no pós-parto de vacas leiteiras. Rev Bras Zootec 30: 1544-1549. doi: 10.1590/ S1516-35982001000600023

20. Lago EP, da Costa AP, Pires A, Susin I, de Farias V, do Lago LA. 2004. Parâmetros metabólicos em vacas leiteiras durante o período de transição pós-parto. Rev Bras Cienc Vet 11: 98103. doi: $10.4322 / \mathrm{rbcv} .2014 .353$

21. Mancuso WA, Marini PR, Dunleavy M. 2016. Peso vivo, condición corporal e indicadores metabólicos en sangre, de vacas lecheras cruza. En: XXXIX Congreso de la Asociación Argentina de Producción Animal. Tandil, Argentina.

22. Manual Merck de Veterinaria. 2000. $5^{\circ}$ ed. Barcelona, España: Océano Grupo Editorial.

23. Margolles E. 1983. Metabolitos sanguíneos en vacas altas productoras durante la gestación-lactancia en las condiciones de Cuba y su relación con trastornos del metabolismo. Rev Cubana Cienc Vet 14: 221-230.

24. Maza L, Vergara O, Álvarez J. 2006. Condición corporal preparto y producción de leche sobrepeso y condición corporal posparto de vacas mestizas. Rev MVZ Córdoba 11: 751-758. doi: 10.21897/rmvz.460
25. Menegoto J, Neto AP, Possa MG, Bernardi F. 2015. Eficiência reprodutiva de vacas leiteiras: escore de condição corporal e periparto resultados parciais. En: V Seminario de Ensino, Pesquisa e Extensão. Brasil.

26. Michailoff A, Agnol DD, Balistieri FS, Mores F, Ferreira R, Bragança JF, Rocha, et al. 2013. Desempenho produtivo e status energético em vacasleiteiras suplementadas com colina protegida. Rev Acad Ciência Anim 11: 367-372. doi: 10.7213/academico.011.004.AO03

27. Minervino AH, Cardoso E, Sá CF, Rodrigues $R$. 2004. Perfil bioquímico sanguíneo de vacas Sindi no pré-parto, pós-parto, início e final de lactação em condições de pastejo na Amazônia. Rev Ciências Agrarias 42: 169-174.

28. Noro M, Borkert J, Hinostroza GA, Pulido R, Wittwer F. 2011. Variaciones diarias de metabolitos sanguíneos y su relación con el comportamiento alimenticio en vacas lecheras a pastoreo primaveral. Rev Cient 21: 125-130.

29. Nozad S, Ramin AG, Moghadam G, Asri-Rezaei S, Babapour A, Ramin S. 2012. Relationship between blood urea, protein, creatinine, triglycerides and macro mineral concentrations with the quality and quantity of milk in dairy Holstein cows. Vet Res Forum 3: 55-59.

30. Oliveira P. 2002. Uso de produtos gliconeogênicos no pré e pós-parto sobre parâmetros produtivos, reprodutivos e sanguíneos de vacas Holandesas. Tese de doutorado. Piracicaba, Sao Paulo, Brasil: Univ. de São Paulo. 120 p.

31. Oliveira RSBR. 2011. Perfil metabólico de vacas mestiças leiteiras uma semana pré-parto e durante o puerpério fisiológico. Tese de mestrado. Minas Gerais, Brasil: Univ. Federal de Uberlândia. 56 p.

32. Penna Junior CO. 2010. Perfil metabólico energético em dois grupos genéticos de vacas holandês $\mathrm{x}$ gir de segunda ordem de parição em dois perío- 
dos da lactação na época da seca nos trópicos. Tese de mestrado. Brasil: Univ. de Federal do Espírito Santo. $64 \mathrm{p}$.

33. Quinteros OR, Vargas JC, Barbona I, Marini PR. 2017. Indicadores metabólicos sanguíneos de genotipos lecheros en pastoreo de la provincia de Napo-Ecuador. La Granja 26: 119-130. doi: 10.17163/lgr.n26.2017.10

34. Ramos L. 2016. Impacto de três manejos reprodutivos na eficiência reprodutiva e econômica de vacas leiteiras em sistema de produção semiintensivo. Tese de Mestrado. Santa Catarina, Brasil: Univ. do Estado de Santa Catarina. $99 \mathrm{p}$.

35. Rennó FP, Pereira JC, Santos ADF, Alves NG, Torres CAA, Rennó LN, et al. 2006. Efeito da condição corporal ao parto sobre a produção e composição do leite, a curva de lactação e a mobilização de reservas corporais em vacas da raça Holandesa. Arq Bras Med Vet Zoo 58: 220-233. doi: 10.1590/S010209352006000200011

36. Roa-Vega ML, Ladino-Romero EA, Hernández-Martínez MC. 2017. Indicadores de bioquímica sanguínea en bovinos suplementados con Cratylia argentea y Saccharomyces cerevisiae. Pastos y Forrajes 40: 144-151.

37. Salgado OR, Vergara OG, Simanca JS. 2008. Relaciones entre peso, condición corporal y producción de leche en vacas del sistema doble propósito. Rev MVZ Córdoba 13: 1360-1364. doi: 10.21897/rmvz.395

38. Santos AD, Rennó F, Alves N, Torres CA, Pereira JC, de Araújo C. 2010. Condição corporal ao parto e produção de leite sobre o desempenho reprodutivo de vacas holandesas em lactação. Rev Bras Saúde Prod Anim 11: 919-931.

39. Saut JPE. 2008. Influência do puerpério e da retenção dos anexos fetais no proteinograma de fêmeas bovinas da raça Holandesa, criadas no Estado de São Paulo. Tese de doutorado. Sao Paulo, Brasil: Univ. de São Paulo. 116 p.

40. Villadiego FAC, Pereira JV, Costa EP, Marcondes MI, Leon VEG, Maitan PP, Nogueira AR, Guimarães JD. 2016. Parâmetros reprodutivos e produtivos em vacas leiteiras de manejo free stall. Pesq Vet Bras 36: 55-61. doi: 10.1590/S0100736X2016000100009

41. Wittwer F. 2000. Diagnóstico dos desequilíbrios metabólicos de energia em rebanhos bovinos. En: González F, Barcellos J, Ospina H, Ribeiro L (eds.) Perfil metabólico em ruminantes: seu uso em nutrição e doenças nutricionais. Porto Alegre, Brasil: UFRGS. 9-22.

42. Wathes DC, Fenwick M, Cheng Z, Bourne N, Llewellyn S, Morris DG, Kenny D, et al. 2007. Influence of negative energy balance on cyclicity and fertility in the high producing dairy cow. Theriogenology 68: 232-241. doi: 10.1016/j.theriogenology.2007.04.006

43. Zárate RF, Pedrozo PR, Contrera JG, Alonso VN, Torres NM, Ortega OP, Lara MN, et al. 2016. Perfil metabólico durante el período de transición preparto y posparto de vacas Holstein del distrito J. Eulogio Estigarribia, Paraguay. Compend Cienc Vet 6: 35-42. doi: $10.18004 /$ compend.cienc.vet. 2016.06.01.35-42 